Proc. Estonian Acad. Sci. Phys. Math., 2001, 50, 1, 5-15

\title{
SUMMATION METHODS OF TRIGONOMETRIC FOURIER SERIES DEFINED BY THE ZAK TRANSFORM
}

\author{
Andi KIVINUKK and Julia VERHOVITŠ \\ Department of Mathematics and Informatics, Tallinn Pedagogical University, Narva mnt. 25, \\ 10120 Tallinn, Estonia; andik@tpu.ee \\ Received 22 March 2000, in revised form 22 August 2000
}

Abstract. We investigate the order of approximation by some new-type summation methods of trigonometric Fourier series. These methods are defined by the Zak transform.

Key words: trigonometric Fourier series, Zak transform, sinc function, modulus of continuity, Lanczos' filter.

\section{INTRODUCTION}

The aim of this paper is to investigate the order of approximation by some new-type summation methods of trigonometric Fourier series. These methods are introduced in $\left.{ }^{1,2}\right]$ for constructing some approximation processes for functions in the disc algebra in uniform norm. The definition of the summation methods is based on the Zak transform.

We introduce some notations. Let $\mathbf{N}, \mathbf{Z}, \mathbf{R}, \mathbf{C}$ denote the sets of all naturals, all integers, all real, and all complex numbers, respectively. Let us consider the triangular $\varphi$-means (or summation methods)

$$
U_{n}(f, x):=\frac{a_{0}}{2}+\sum_{k=1}^{n} \varphi\left(\frac{k}{n}\right)\left(a_{k} \cos k x+b_{k} \sin k x\right)
$$

of the real Fourier series of a $2 \pi$-periodic continuous function $f \in C_{2 \pi}$ with Fourier coefficients $a_{k}, b_{k}$. Several $\varphi$-means can be defined by the continuous 
function $\varphi \in C_{[0,1]}$, which satisfies the boundary conditions $\varphi(0)=1, \varphi(1)=0$. Our new summation methods are constructed as follows. The Zak transform $Z \psi: \mathbf{R} \times \mathbf{R} \rightarrow \mathbf{C}$ (cf. $\left[{ }^{3}\right]$, pp. 164,$166 ;\left[^{4}\right]$, pp. 109-110) of a function $\psi: \mathbf{R} \rightarrow \mathbf{C}$ is formally defined by

$$
Z \psi(u, v):=\sum_{l \in \mathbf{Z}} \psi(u+l) e^{i l v} \quad(u, v \in \mathbf{R}) .
$$

Since $Z \psi$ is a quasi-periodic function, i.e.

$$
Z \psi(u+1, v)=e^{-i v} Z \psi(u, v), \quad Z \psi(u, v+2 \pi)=Z \psi(u, v) \quad(u, v \in \mathbf{R}),
$$

it is reasonable to consider the variables $(u, v) \in[0,1] \times \mathbf{R}$ only. We are interested in the Zak transform of a real-valued and even function $\theta$. Then it will be convenient to define

$$
Z \theta(u, v):=\sum_{l \in \mathbf{Z}} \theta(u+l) \cos l v \quad(u \in[0,1], v \in \mathbf{R})
$$

Obviously, the (real even) Zak transform (2) of an even function is even in both variables $u$ and $v$. The simplest condition for the Zak transform $Z \theta$ to be welldefined is

$$
\sum_{l \in \mathbf{Z}}|\theta(u+l)|<\infty
$$

Actually, we shall use only one part of the Zak transform defined by

$$
Z^{+} \theta(u, v):=\sum_{l=0}^{\infty} \theta(u+l) \cos l v \quad(u \in[0,1], v \in \mathbf{R}) .
$$

Now our new-type summation method $U_{n}^{z}\left(\left[^{1}\right]\right.$, p. 312) is defined by (1), where

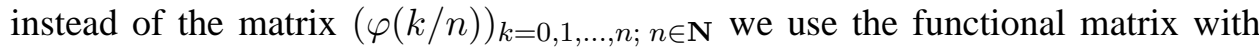
entries

$$
\Phi\left(\frac{k}{n}, n x\right):=Z^{+} \theta\left(\frac{k}{n}, n x\right) \quad(k=0,1, \ldots, n ; n \in \mathbf{N}, x \in \mathbf{R}),
$$

explicitly,

$$
U_{n}^{z}(f, x):=\frac{a_{0}}{2}+\sum_{k=1}^{n} \Phi\left(\frac{k}{n}, n x\right)\left(a_{k} \cos k x+b_{k} \sin k x\right) .
$$

First we remark that $U_{n}^{z}$ is non-polynomial, i.e. in contrast with (1), $U_{n}^{z} f$ is not a trigonometric polynomial. Denote

$$
A_{0}(f, x):=a_{0} / 2, \quad A_{k}(f, x):=a_{k} \cos k x+b_{k} \sin k x \quad(k \in \mathbf{N}) .
$$

6 
Then, under the condition (3) we may write

$$
U_{n}^{z}(f, x)=\sum_{l=0}^{\infty}\left(\sum_{k=0}^{n} \theta\left(l+\frac{k}{n}\right) A_{k}(f, x)\right) \cos \ln x .
$$

Now it is clear that $U_{n}^{z}$ forms linear transformation on $C_{2 \pi}$ into $C_{2 \pi}$. We also see that the first summand of $U_{n}^{z}$ in (8) is the ordinary summation method (1) defined by $\theta$.

We must choose the function $\theta$ so that, like for $\varphi$ in (1), $\Phi(0, v)=1$ and $\Phi(1, v)=0$ for all $v \in \mathbf{R}$. Moreover, under some additional conditions on $\theta$ we shall find the order of approximation by the method (6). It turns out that the approximation properties of $U_{n}^{z}$ depend on the Fourier-cosine transform of $\theta$ on a discrete set of points. To understand the situation better, we recall an earlier result $\left[{ }^{5}\right]$ which we call the subordination principle via the Rogosinski-type summation methods.

\section{SUBORDINATION PRINCIPLE VIA ROGOSINSKI-TYPE SUMMATION METHODS}

The Rogosinski-type summation methods (or means), denoted by $R_{n, j}$, were introduced by Rogosinski $\left[{ }^{6}\right]$ in the form $(j \in \mathbf{N})$

$$
R_{n, j}(f, x):=\frac{a_{0}}{2}+\sum_{k=1}^{n} \cos \left(\left(j-\frac{1}{2}\right) \frac{k \pi}{n}\right)\left(a_{k} \cos k x+b_{k} \sin k x\right) .
$$

It is remarkable that here the generating functions $\varphi_{j}(t):=\cos \left(j-\frac{1}{2}\right) \pi t$ $(j \in \mathbf{N})$ constitute an orthogonal system $\left\{\varphi_{j}\right\}$ on $[0,1]$ and the boundary conditions $\varphi_{j}(0)=1, \varphi_{j}(1)=0$ are valid for all $j \in \mathbf{N}$. This circumstance inspired us to represent the function (5) by a Fourier series using the system $\left\{\varphi_{j}\right\}$. To simplify the notations, let $m_{j}:=\left(j-\frac{1}{2}\right) \pi$. Let us evaluate the Fourier coefficients of $\Phi$ by the system $\left\{\varphi_{j}\right\}$. We have

$$
d_{j}(v):=2 \int_{0}^{1} \Phi(u, v) \cos \left(m_{j} u\right) d u
$$

and formally

$$
\Phi(u, v)=\sum_{j=1}^{\infty} d_{j}(v) \varphi_{j}(u) .
$$

Now our subordination principle (see $\left[{ }^{5}\right]$, Theorem 1') reads as follows.

Theorem A. Let the sequence of coefficients (10) satisfy

$$
\sum_{j=1}^{\infty}\left|d_{j}(v)\right|<\infty
$$


where the series is converging uniformly for $v \in \mathbf{R}$. If the function $\Phi$ in (5) generates the summation method $U_{n}^{z}$ by (6), then we have

$$
\begin{gathered}
U_{n}^{z}(f, x)=\sum_{j=1}^{\infty} d_{j}(n x) R_{n, j}(f, x), \\
f(x)-U_{n}^{z}(f, x)=\sum_{j=1}^{\infty} d_{j}(n x)\left(f(x)-R_{n, j}(f, x)\right) .
\end{gathered}
$$

By Theorem A we see that the generalized Rogosinski means are very useful for the investigation of methods $U_{n}^{z}$. Therefore we continue with a theorem for the generalized Rogosinski means $\left.R_{n, j}{ }^{5}\right]$. Let $\omega_{k}(f, \delta)$ be the $k$ th modulus of continuity (see $\left[{ }^{7}\right]$ ) of the function $f \in C_{2 \pi}$.

Theorem B. For all $j \in \mathbf{N}$

$$
\sup _{n \in \mathbf{N}}\left\|R_{n, j}\right\|=\frac{4}{\pi^{2}} \log j+O(1) .
$$

Moreover, there exist two positive absolute constants $M_{q}(q=1$ or $q=2)$ such that the generalized Rogosinski means have the order of approximation

$$
\left\|f-R_{n, j} f\right\|_{C_{2 \pi}} \leq M_{q} j^{q} \omega_{q}\left(f, \frac{1}{n}\right)
$$

for $q=1$ or $q=2$, respectively.

As a straightforward consequence of Theorems A and B we formulate two theorems (cf. $\left.\left[{ }^{5}\right]\right)$ that we shall apply to the new summation method $U_{n}^{z}$ introduced in $\left[{ }^{1}\right]$.

Theorem 1. Let for the sequence of coefficients in (10) there exist an absolute (independent of $v \in \mathbf{R}$ ) constant $m_{0}$ such that

$$
\sum_{j=1}^{\infty}\left|d_{j}(v)\right| \log j \leq m_{0}<\infty .
$$

If the function $\Phi$ in (5) generates the summation method $U_{n}^{z}$ by (6), then $U_{n}^{z}$ forms a uniformly bounded linear transformation on $C_{2 \pi}$ into $C_{2 \pi}$.

Theorem 2. Let for the sequence of coefficients in (10) there exist an absolute (independent of $v \in \mathbf{R})$ constant $m_{q}(q=1$ or $q=2)$ such that

$$
\sum_{j=1}^{\infty} j^{q}\left|d_{j}(v)\right| \leq m_{q}<\infty .
$$

Then, for any $f \in C_{2 \pi}$, we have the order of approximation

$$
\left\|f-U_{n}^{z} f\right\|_{C_{2 \pi}} \leq M_{q} m_{q} \omega_{q}\left(f, \frac{1}{n}\right),
$$

where $q=1$ or $q=2$, respectively, and the constants $M_{q}, m_{q}$ are from Theorem $B$ and from (15), respectively. 


\section{APPLICATIONS TO SOME EXAMPLES OF APPROXIMATION METHODS}

We are looking for some examples of the function $\theta$ in (5) so that the condition (15) would be valid.

Example 1. One of the simplest choices for $\theta$ is the sinc function defined by the equality

$$
\operatorname{sinc} x:=\left\{\begin{array}{cc}
\frac{\sin \pi x}{\pi x}, & x \neq 0 \\
1, & x=0 .
\end{array}\right.
$$

Since $\operatorname{sinc} l=0$ for $l \in \mathbf{Z} \backslash\{0\}$, it is clear that for

$$
\Phi_{1}(u, v):=Z^{+} \operatorname{sinc}(u, v)
$$

in (5) we have

$$
\Phi_{1}(0, v)=1, \quad \Phi_{1}(1, v)=0 \quad(v \in \mathbf{R}) .
$$

Unfortunately, the sinc function decreases too slowly to quarantee that the condition (15) is satisfied. But here is another interesting feature.

First, we can find the Zak transform (2) explicitly, i.e.

$$
Z \operatorname{sinc}(u, v)=\sum_{l \in \mathbf{Z}} \operatorname{sinc}(u+l) \cos l v=\sum_{l \in \mathbf{Z}} \operatorname{sinc}(u-l) \cos l v=\cos (u v)
$$

for $u \in \mathbf{R}, v \in(-\pi, \pi)$ (see $\left[{ }^{8}\right]$, pp. 62-63). Here the last equality says that the Zak transform of the sinc function is the Fourier series of the function $\cos (u v)$. This case is interesting also because here the Zak transform does exist, but the condition (3) is not valid.

Second, in the case of the sinc function the first summand in (8) is the famous Lanczos' filter (see, e.g., $\left[{ }^{9}\right]$, Ch. 22, Sec. 22.6). We shall consider the Lanczos' filter in our last section.

The function $\operatorname{sinc}^{2} x$ has better decay than $\operatorname{sinc} x$ and preserves all nice properties of $\operatorname{sinc} x$. Therefore, in the following we shall use $\operatorname{sinc}^{2} x$.

Example 2. Let the summation method $U_{n}^{z, 2}$ be defined by (6), where instead of $\Phi$ we have

$$
\Phi_{2}(u, v):=Z^{+} \operatorname{sinc}^{2}(u, v) .
$$

Obviously, the conditions (16) are valid also for $\Phi_{2}$. We need some technical lemmas to check (15). Since by (4)

$$
\Phi_{2}(u, v)=\sum_{l=0}^{\infty} \operatorname{sinc}^{2}(u+l) \cos l v \quad(u \in[0,1], v \in \mathbf{R}),
$$


we first find estimations for shifted Fourier-cosine transform of $\operatorname{sinc}^{2} x$, i.e. for

$$
\delta_{l, j}:=\int_{0}^{1} \operatorname{sinc}^{2}(l+t) \cos \left(m_{j} t\right) d t \quad(l=0,1, \ldots ; \quad j \in \mathbf{N}) .
$$

As before, $m_{j}:=\left(j-\frac{1}{2}\right) \pi$. Integration by parts in (17) gives

Lemma 1. Let $g$ be r-times differentiable on $[0,1]$. Then

$$
\begin{aligned}
\int_{0}^{1} g(t) \cos (a t) d t= & (-1)^{r} \frac{1}{a^{r}} \int_{0}^{1} g^{(r)}(t) \cos \left(a t-\frac{\pi r}{2}\right) d t \\
& +\left.\sum_{k=0}^{r-1}(-1)^{k} \frac{1}{a^{k+1}} g^{(k)}(t) \cos \left(a t-\frac{\pi(k+1)}{2}\right)\right|_{0} ^{1} .
\end{aligned}
$$

To simplify notations, let

$$
s_{k}(t):=\operatorname{sinc}^{k} t \quad(k=1,2,3,4) .
$$

To use Lemma 1, we must evaluate several derivatives.

Lemma 2. We have

$$
s_{1}^{\prime}(0)=s_{1}^{\prime \prime \prime}(0)=0, \quad s_{1}^{\prime \prime}(0)=-\pi^{2} / 3, \quad s_{1}^{(4)}(0)=\pi^{4} / 5,
$$

and for $l \in \mathbf{N}$

$$
\begin{gathered}
s_{1}(l)=0, \quad s_{1}^{\prime}(l)=\frac{(-1)^{l}}{l}, \quad s_{1}^{\prime \prime}(l)=\frac{(-1)^{l+1} 2}{l^{2}} \\
s_{1}^{\prime \prime \prime}(l)=\frac{(-1)^{l+1}}{l}\left(\pi^{2}-\frac{6}{l^{2}}\right), \quad s_{1}^{(4)}(l)=\frac{(-1)^{l} 4}{l^{2}}\left(\pi^{2}-\frac{6}{l^{2}}\right) .
\end{gathered}
$$

Proof. We get the derivatives in (19) from the power series of the sinc function. For (20) we write

$$
\pi t s_{1}(t)=\sin \pi t,
$$

which by Leibniz's rule for the $n$th derivative yields

$$
t s_{1}^{(n)}(t)+n s_{1}^{(n-1)}(t)=\pi^{n-1} \sin (\pi t+n \pi / 2) .
$$

Putting $t=l \in \mathbf{N}$, we get step by step the equalities (20).

Lemma 3. For all $l=0,1, \ldots ; \quad j \in \mathbf{N}$ the shifted cosine transform of $\operatorname{sinc}^{2} t$ in (17) has the estimation

$$
\delta_{l, j}=O\left((l+1)^{-2} j^{-3}\right) .
$$


Proof. If in Lemma 1 we take $g(t)=s_{2}(l+t), r=3, a=m_{j}:=(j-1 / 2) \pi$, then we obtain

$$
\delta_{l, j}=-\frac{s_{2}^{\prime}(l)}{m_{j}^{2}}+(-1)^{j} \frac{s_{2}^{\prime \prime}(l+1)}{m_{j}{ }^{3}}+\frac{1}{m_{j}{ }^{3}} \int_{0}^{1} s_{2}^{\prime \prime \prime}(l+t) \sin m_{j} t d t
$$

Since by (18) $s_{2}=s_{1}^{2}$, we get

$$
s_{2}^{\prime}=2 s_{1} s_{1}^{\prime}, \quad s_{2}^{\prime \prime}=2\left(s_{1}^{\prime}\right)^{2}+2 s_{1} s_{1}^{\prime \prime}, \quad s_{2}^{\prime \prime \prime}=6 s_{1}^{\prime} s_{1}^{\prime \prime}+2 s_{1} s_{1}^{\prime \prime \prime} .
$$

Now (20) yields for $l \in \mathbf{N}$

$$
s_{2}(l)=s_{2}^{\prime}(l)=0, \quad s_{2}^{\prime \prime}(l)=\frac{2}{l^{2}},
$$

and for (23) we may write

$$
\delta_{l, j}=\frac{(-1)^{j} 2}{(l+1)^{2} m_{j}{ }^{3}}+\frac{1}{m_{j}{ }^{3}} \int_{0}^{1} s_{2}^{\prime \prime \prime}(l+t) \sin m_{j} t d t .
$$

Since by definition (18) $s_{1}(t)=O\left(t^{-1}\right) \quad(t>0)$, it follows from (22) that $s_{1}^{\prime}(t)=O\left(t^{-1}\right), \quad s_{1}^{\prime \prime}(t)=O\left(t^{-1}\right), \quad s_{1}^{\prime \prime \prime}(t)=O\left(t^{-1}\right) \quad(t \geq 1)$. Therefore, by (24) $s_{2}^{\prime \prime \prime}(t)=O\left(t^{-2}\right) \quad(t \geq 1)$ and for (26) we have for all $l \in \mathbf{N}$

$$
\left|\delta_{l, j}\right| \leq \frac{2}{(l+1)^{2} m_{j}{ }^{3}}+\frac{M}{m_{j}{ }^{3}} \int_{0}^{1} \frac{d t}{(l+t)^{2}}=O\left((l+1)^{-2} j^{-3}\right),
$$

as $m_{j}=(j-1 / 2) \pi$. Since by (24) and by Lemma $2 s_{2}^{\prime \prime \prime}$ is bounded on $[0, \infty)$, the equality (26) implies $\delta_{0, j}=O\left(j^{-3}\right)$. The proof is complete.

Now it is easy to check [see (10), (5), (4), and (17)] that for

$$
\begin{aligned}
d_{j, 2}(v): & =2 \int_{0}^{1} Z^{+} \operatorname{sinc}^{2}(u+v) \cos \left(m_{j} u\right) d u \\
& =2 \sum_{l=0}^{\infty} \cos l v \int_{0}^{1} \operatorname{sinc}^{2}(u+l) \cos \left(m_{j} u\right) d u
\end{aligned}
$$

Lemma 3 yields

$$
d_{j, 2}(v)=O\left(j^{-3}\right) \quad(j \in \mathbf{N}, v \in \mathbf{R}) .
$$

Therefore, the condition (15) is valid for $q=1$ and by Theorem 2 we obtain as follows. 
Theorem 3. The summation method

$$
U_{n}^{z, 2}(f, x)=\sum_{l=0}^{\infty}\left(\sum_{k=0}^{n} \operatorname{sinc}^{2}\left(l+\frac{k}{n}\right) A_{k}(f, x)\right) \cos \ln x,
$$

generated by the Zak transform of the sinc ${ }^{2}$ function, forms a uniformly bounded linear transformation on $C_{2 \pi}$ into $C_{2 \pi}$. Moreover, there exists an absolute constant $M>0$ such that for all $f \in C_{2 \pi}$

$$
\left\|f-U_{n}^{z, 2} f\right\|_{C_{2 \pi}} \leq M \omega_{1}\left(f, \frac{1}{n}\right) .
$$

Proceeding from Example 2, in the following we could attempt with $\operatorname{sinc}^{3} t$. In this case we will have the same order of approximation as in Theorem 3 (see $\left[{ }^{10}\right]$ ). This observation fits well with the Fourier-cosine transform of the function $\varphi$ in the definition of ordinary summation methods (1). Let us define the Fourier-cosine transform by

$$
\varphi^{\wedge}(s):=\int_{0}^{1} \varphi(u) \cos (\pi s u) d u .
$$

Then, for $\varphi_{1}(t):=1$ (Fourier partial sums) we have $\varphi_{1}^{\wedge}(s)=\operatorname{sinc} s$, for $\varphi_{2}(t):=$ $1-t$ (Fejér means) we have $\varphi_{2}^{\wedge}(s)=\frac{1}{2} \operatorname{sinc}^{2}(s / 2)$, for $\varphi_{3}(t):=1-6 t^{2}+6 t^{3}$ if $0 \leq t \leq \frac{1}{2}$ and $\varphi_{3}(t):=2(1-t)^{3}$ if $\frac{1}{2} \leq t \leq 1$ (Jackson-de La Vallée Poussin means) we have $\varphi_{3}^{\wedge}(s)=\frac{3}{8} \operatorname{sinc}^{4}(s / 4)$. It is known that in space $C_{2 \pi}$ the Fourier partial sums may diverge, but the Fejér means and the Jackson-de La Vallée Poussin means have the order of approximation $\omega_{2}(f, 1 / \sqrt{n})$ and $\omega_{2}(f, 1 / n)$, respectively (see $\left[{ }^{11}\right]$, pp. 77, 205). Let us remark that we can find this order of approximation by the Jackson-de La Vallée Poussin means also by using Theorem 2. Indeed, here $d_{j}=2 \varphi_{3}^{\wedge}\left(m_{j}\right)=O\left(j^{-4}\right)$ and the condition (15) is valid with $q=2$. After this discussion we continue with $\operatorname{sinc}^{4} t$.

Example 3. Let the summation method $U_{n}^{z, 4}$ be defined by (6), where instead of $\Phi$ we have

$$
\Phi_{4}(u, v):=Z^{+} \operatorname{sinc}^{4}(u, v) .
$$

Obviously, the conditions (16) are valid also for $\Phi_{4}$. This example is quite similar to Example 2. Therefore we only sketch the proofs. We deduce now from Lemma 1 that

$$
\begin{aligned}
\delta_{l, j}= & \frac{(-1)^{j+1}}{m_{j}} s_{4}(l+1)-\frac{s_{4}^{\prime}(l)}{m_{j}{ }^{2}}+(-1)^{j} \frac{s_{4}^{\prime \prime}(l+1)}{m_{j}{ }^{3}}+\frac{s_{4}^{\prime \prime \prime}(l)}{m_{j}{ }^{4}} \\
& +\frac{1}{m_{j}{ }^{4}} \int_{0}^{1} s_{4}^{(4)}(l+t) \cos \left(m_{j} t\right) d t .
\end{aligned}
$$


If in (24) we replace $s_{1}$ by $s_{2}$ and $s_{2}$ by $s_{4}$, then we have by (25) for all $l \in \mathbf{N}$

$$
s_{4}(l)=s_{4}^{\prime}(l)=s_{4}^{\prime \prime}(l)=s_{4}^{\prime \prime \prime}(l)=0 .
$$

This already yields

$$
\delta_{l, j}=\frac{1}{m_{j}{ }^{4}} \int_{0}^{1} s_{4}^{(4)}(l+t) \cos \left(m_{j} t\right) d t
$$

Using

$$
s_{4}^{(4)}=6\left(s_{2}^{\prime \prime}\right)^{2}+8 s_{2}^{\prime} s_{2}^{\prime \prime \prime}+2 s_{2} s_{2}^{(4)}
$$

gives step by step the estimation $s_{4}^{(4)}(x)=O\left(x^{-4}\right)$ for all $x \geq 1$. Hence,

$$
\delta_{l, j}=O\left((l+1)^{-4} j^{-4}\right)
$$

and for

$$
\begin{aligned}
d_{j, 4}(v) & :=2 \int_{0}^{1} Z^{+} \operatorname{sinc}^{4}(u+v) \cos \left(m_{j} u\right) d u \\
& =2 \sum_{l=0}^{\infty} \cos l v \int_{0}^{1} \operatorname{sinc}^{4}(u+l) \cos \left(m_{j} u\right) d u
\end{aligned}
$$

it follows that

$$
d_{j, 4}(v)=O\left(j^{-4}\right) \quad(j \in \mathbf{N}, v \in \mathbf{R}) .
$$

The condition (15) is fulfilled for $q=2$ and by Theorem 2 we obtain as follows.

Theorem 4. The summation method

$$
U_{n}^{z, 4}(f, x):=\sum_{l=0}^{\infty}\left(\sum_{k=0}^{n} \operatorname{sinc}^{4}\left(l+\frac{k}{n}\right) A_{k}(f, x)\right) \cos \ln x,
$$

generated by the Zak transform of the sinc ${ }^{4}$ function, forms a uniformly bounded linear transformation on $C_{2 \pi}$ into $C_{2 \pi}$. Moreover, there exists an absolute constant $M>0$ such that for all $f \in C_{2 \pi}$

$$
\left\|f-U_{n}^{z, 4} f\right\|_{C_{2 \pi}} \leq M \omega_{2}\left(f, \frac{1}{n}\right)
$$




\section{LANCZOS' FILTER}

Lanczos' filter is important for reducing the effect of the Gibbs phenomenon (see $\left[{ }^{9,12}\right]$ ). In $\left[{ }^{13}\right]$, pp. 332-333, we find the order of approximation by Lanczos' method, defined by

$$
L_{n}(f, x):=\frac{a_{0}}{2}+\sum_{k=1}^{n} \operatorname{sinc}\left(\frac{k}{n}\right)\left(a_{k} \cos k x+b_{k} \sin k x\right),
$$

as follows:

$$
\left\|f-L_{n} f\right\|_{C_{2 \pi}} \leq 3 \rho\left(f, \frac{\pi}{n}\right)
$$

Here

$$
\rho(f, h):=\frac{1}{2 h}\left\|\int_{-h}^{h}|f(\cdot+t)-f(\cdot)| d t\right\|_{C_{2 \pi}} \quad(h>0) .
$$

So far we have not found any references to the order of approximation by $L_{n}$ via the modulus of continuity. We present here a simple order of approximation by $L_{n}$. For the consideration we need our earlier result (here only a special case is given) as follows $\left(\left[{ }^{14}\right]\right.$; cf. also $\left[{ }^{13}\right]$, p. 312$)$.

Theorem C. Let the summation method $U_{n}$ be defined by (1), where the function $\varphi$ has for some $r \in \mathbf{N}$ the representation

$$
\varphi(u)=1-\sum_{j=r}^{\infty} c_{j} u^{2 j}, \quad \sum_{j=r}^{\infty}\left|c_{j}\right|<\infty .
$$

Then there exists a constant $M(r)>0$ such that

$$
\left\|f-U_{n} f\right\|_{C_{2 \pi}} \leq M(r) \omega_{2 r}\left(f, \frac{1}{n}\right)
$$

for all $f \in C_{2 \pi}$.

The condition (31) is valid for the sinc function due to the expansion

$$
\operatorname{sinc}(u)=1-\sum_{j=1}^{\infty}(-1)^{j+1} \frac{\pi^{2 j}}{(2 j+1) !} u^{2 j} .
$$

Now by Theorem $\mathrm{C}$ we have the order of approximation of Lanczos' method.

Theorem 5. For some $M>0$ and for all $f \in C_{2 \pi}$ we have the estimation

$$
\left\|f-L_{n} f\right\|_{C_{2 \pi}} \leq M \omega_{2}\left(f, \frac{1}{n}\right) .
$$




\section{ACKNOWLEDGEMENT}

This work was partially supported by the Estonian Science Foundation (grant No. 3620).

\section{REFERENCES}

1. Schipp, F. and Szili, L. Approximation in $H^{\infty}$-norm. In Approximation Theory and Function Series (Vértesi, P., ed.). Bolyai Soc. Math. Stud., 1996, 5, 307-320.

2. Bokor, J., Schipp, F. and Gianone, L. Approximate $H^{\infty}$ identification using partial sum operators in the disc algebra basis. In Proceedings of the American Control Conference, Seattle WA, 1995, 1981-1985.

3. Benedetto, J. Harmonic Analysis and Applications. CRC Press, Boca Raton, 1997.

4. Daubechies, I. Ten Lectures on Wavelets. SIAM, Philadelphia, 1992.

5. Kivinukk, A. Subordination in Fourier approximation by generalized Rogosinski means and its applications. In Approximation Theory and Function Series (Vértesi, P., ed.). Bolyai Soc. Math. Stud., 1996, 5, 237-245.

6. Rogosinski, W. Reihensummierung durch Abschnittskoppelungen. Math. Z., 1926, 25, $132-149$

7. Timan, A. F. Theory of Approximation of Functions of a Real Variable. MacMillan, New York, 1963 (Russian original: Fizmatgiz, Moscow, 1960).

8. Higgins, J. R. Sampling Theory in Fourier and Signal Analysis: Foundations. Clarendon Press, Oxford, 1996.

9. Hamming, R. W. Numerical Methods for Scientists and Engineers. McGraw-Hill, New York, 1962 (Russian edn.: Nauka, Moscow, 1972).

10. Verhovitš, J. Order of approximation by some new-type summation methods of Fourier series. B.Sc. thesis, Tallinn Pedagogical Univ., Tallinn, 1998 (in Russian).

11. Butzer, P. L. and Nessel, R. J. Fourier Analysis and Approximation. Birkhäuser, Basel, 1971.

12. Gottlieb, D. and Shu, C.-W. On the Gibbs phenomenon and its resolution. SIAM Rev., 1997, 39, 644-668.

13. Zhuk, V. V. Approximation of Periodic Functions. Izd. LGU, Leningrad, 1982 (in Russian).

14. Kivinukk, A. Comparison theorems for summation processes of Fourier expansions in Banach spaces. Acta Comment. Univ. Tartuensis, 1978, 448, 31-39 (in Russian).

\section{ZAKI TEISENDUSEGA DEFINEERITUD TRIGONOMEETRILISTE FOURIER' RIDADE SUMMEERIMISMEETODID}

On leitud Zaki teisendusega defineeritud trigonomeetriliste Fourier' ridade summeerimismeetodite koonduvuskiirused. Nimetatud meetodid on võetud kasutusele artiklis $\left[{ }^{1}\right]$, kus neid rakendati teatud funktsioonialgebra baasi konstrueerimiseks. 